\title{
The Red Blood Cell Distribution Width-Albumin Ratio Was a Potential Prognostic Biomarker for Diabetic Ketoacidosis
}

\section{Depu Zhou \\ Jie Wang (D) \\ Xiaokun Li}

Department of Endocrinology, Yanbian University Hospital, Yanji, Jilin Province, People's Republic of China
Correspondence: Xiaokun $\mathrm{Li}$ Department of Endocrinology, Yanbian University Hospital, No. I 19, Shizi Street, Yanji City, Jilin Province, 133000, People's Republic of China

Tel +86 433-2660l70

Email professerlxk@tom.com
Background: The red blood cell distribution width (RDW)-albumin ratio (RA) is a new biomarker, which is d-efined as RDW divided by albumin. This study aimed at determining the prognostic values of RA for diabetic ketoacidosis (DKA).

Methods: Data were obtained from Medical Information Mart for Intensive Care Database III V1.4 (MIMIC-III) and the RA calculated. Multivariate Cox regression analysis was performed to determine the correlation between RA and 90-day mortality or 365-day mortality. To further investigate the association with RA and mortality, the patients were divided into two groups. The second outcome was the association between the incidence of DKA-related infections and RA.

Results: For DKA patients in the ICU, RA was significantly correlated with 90-day mortality (HR: 2.1, 95\% CI: 1.5, 3.0, p < 0.001) and 365-day mortality (HR: 1.9, 95\% CI: $1.5,2.5, \mathrm{p}<0.001)$. A high RA was independently correlated with increased 90 -day mortality (HR: 7.8, 95\% CI: 1.8, 34.0, p for trend $<0.001$ ) and 365-day mortality (HR: $5.2,95 \% \mathrm{CI}$ : $2.4,11.3, \mathrm{p}$ for trend $<0.001)$. Moreover, RA was found to be an independent predictor for sepsis and septic shock in patients with DKA (HR: 2.9, 95\% CI: 2.0, 4.1, p<0.001). After adjusting for confounders, the statistical outcome was the same.

Conclusion: A high RA is significantly correlated with increased all-cause mortality of DKA as well as an increased incidence of DKA-related infections. RA is a potential prognostic marker for DKA.

Keywords: red blood cell distribution width-albumin ratio, diabetic ketoacidosis, diabetic ketoacidosis-associated infection, all-cause mortality

\section{Introduction}

Diabetic ketoacidosis (DKA) is an acute life-threatening complication of diabetes mellitus (DM). The diagnostic criteria of DKA involve hyperglycaemia due to insulin deficiency, positive ketone tests, and metabolic acidosis. ${ }^{1}$ While there are about $1-7 \%$ of DKA patients with absence of hyperglycemia (blood glucose $<250 \mathrm{mg} / \mathrm{dl}$ ). ${ }^{2}$ With the increasing global incidence of DM, the prevalence of DKA in DM patients has also increased. ${ }^{3,4}$ Among DM patients, about $4-9 \%$ of all acute hospital admissions and $3.5-4.5 \%$ of mortalities are associated with DKA. ${ }^{5}$ Due to these high rates, it is important to establish an early biomarker for predicting the prognosis of DKA patients.

Even though the mechanisms through which DKA progresses have not been established, several studies suggest that inflammation may be the leading factor 
promoting the progression of DKA. ${ }^{6-8}$ Hyperglycemia induces systemic inflammation, enhances cytokine secretion, reduces the secretion and action of insulin, and elevates the generation of free fatty acids, which are ketogenic substrates. Meanwhile, ketosis-induced high oxidative stress levels may lead to abnormal glucose uptake, insulin resistance and decreased insulin secretion. ${ }^{9}$ Red blood cell distribution width (RDW), which reflects the heterogeneity of red blood cell (RBC) sizes, is calculated using standard deviation volumes and mean corpuscular volumes of erythrocytes. A high RDW is associated with systemic inflammation and is a predictor for the poor prognoses of many diseases, including cardiovascular, kidney, diabetes, liver and respiratory diseases. ${ }^{10}$ Albumin is a major substance maintaining plasma osmolarity before researchers find the association between it and inflammation. ${ }^{11,12} \mathrm{~A}$ low albumin level has been shown to be a marker for poor outcomes in patients with malignancies, critical illnesses, and thrombotic diseases. ${ }^{11}$

Although the roles of RDW or albumin in DKA patients have been reported, ${ }^{13,14}$ it is important to establish the prognostic value of RA in these patients. The association between RA and DKA has not been documented. Therefore, we aimed at investigating the association between RA and clinical outcomes of DKA and DKArelated infections.

\section{Methods}

\section{Data Source}

The relevant patient data was downloaded from the Medical Information Mart for Intensive Care Database III V1.4 (MIMIC-III). The database contains private medical records for 53,423 patients who were in the intensive care units (ICU) of Beth Israel Deaconess Medical Center (Boston, USA) from 2001 to 2012. Beth Israel Deaconess Medical Center is a public tertiary care hospital. The data in this database include that of hourly vital signs, laboratory results, procedures, prescriptions, and diagnostic codes. To get access to the database, we completed the course "Protecting Human Research Participants" at the website of the National Institutes of Health and obtained the certification. This study was approved by the Massachusetts Institute of Technology and the Institutional Review Boards. All data accessed complies with relevant data protection and privacy regulations.

\section{Participant Selection Criteria}

DKA patients were selected according to the international classification of disease codes-9 (ICD-9). The inclusion criteria were as follows: 1) age $<18$ years; 2) Stay time in ICU $>48$ hours; 3 ) Those without hematologic diseases; 4) those with comprehensive RDW and serum albumin data; and 5) those whose missing data $<5 \%$.

\section{Data Extraction and Outcomes}

The data of DKA patients from MIMIC-III (V1.4) were extracted by structure query language. All the data we collected included age, grand, heart rate, respiratory rate, temperature, percutaneous oxygen saturation (SPO2) systolic blood pressure (SBP), diastolic blood pressure (DBP), serum albumin, lactate, white blood cell (WBC), congestive heart failure (CHF), valvular disease, renal failure, chronic pulmonary disease, hypothyroidism, liver disease, sepsis, sequential organ failure assessment score (SOFA), simplified acute physiology score II (SAPS II).

We enrolled 375 DKA patients from MIMIC-III (V1.4). All the patients were divided into two groups according to the value of RA: RA $<3.88$ was regarded as low group, while the others were regarded as high group. All-cause mortality was the primary outcome. Since DKA patients were the most susceptible for infection, we chose sepsis as the secondary outcome.

\section{Statistical Analysis}

Continuous variables were expressed as mean \pm standard deviation or interquartile range (IQR), and statistical differences between two groups were accessed by KruskalWallis test. While categorical data were expressed as frequencies, and the $\chi^{2}$ test or Fisher's exact test (expected frequency $<10$ ) was used to compare the differences between groups.

Multivariate Cox regression models were performed to estimate the association between RA and all-cause mortality in DKA. The relationship between RA and DKA-related sepsis was assessed by the Cox proportional hazard regressions. To further accurate the relationships, the patients were divided into seconde according to value of RA. The outcomes of Cox regression were presented as hazard ratios (HRs) with 95\% confidence intervals (CIs). Model I was adjusted for the confounders age, gender. Model II was adjusted for age, gender, anion gap, CHF, liver disease.

A p $<0.05$ was considered statistically significant and $\mathrm{p}<0.01$ highly statistically significant. $\mathrm{R}$ software 
(Version 3.6.2, http://www.r-project.org) was used to conduct analyses.

\section{Results}

\section{Characteristics of DKA Patients}

We enrolled a total of 375 participants and grouped them into two groups: RA $<3.88$ was regarded as the low group, while RA $>3.88$ was considered as the high group (Table 1). DKA patients with high RA tended to be older, had low levels of heart rates, respiratory rates and DBP. They were more likely to exhibit high levels of SAPSII, SOFA, and had comorbidities of CHF, valvular disease, renal failure, and sepsis.

\section{The Association Between RA and All-Cause Mortality of DKA Patients in the ICU}

Patients in the high RA group exhibited a higher mortality rate than those in the low RA group (Table 1). To investigate the association between RA and mortality, we performed the Cox regression analysis and the results are shown in Table 2. RA was found to be an independent predictor for 90-day mortality (HR: $2.1,95 \%$ CI: 1.5, 3.0, $\mathrm{p}<0.001$ ) and 365-day mortality (HR: 1.9, 95\% CI: 1.5, 2.5, p<0.001) in DKA patients. After adjusting for age and gender, RA was independently associated with 90-day (HR: $1.8,95 \%$ CI: 1.3, 2.6, $\mathrm{p}<0.001$ ) and 365-day mortality (HR: 1.6, 95\% CI: 1.3, 2.1, $\mathrm{p}<0.001)$. After adjusting for multiple confounders, RA was still found to exhibit a statistical significance for 90-day (HR: 2.2, 95\% CI: 1.4, 3.6, p < 0.001) and 365-day mortality (HR: 1.6, 95\% CI: 1.2, 2.1, $\mathrm{p}<0.001)$. Moreover, second-order analysis also revealed that high RA levels are associated with increased mortality rates among DKA patients. Compared to the reference, the HRs and $95 \%$ CIs of RA for the 90-day mortality rate were $7.8(1.8,34.0)$ in the nonadjusted model, $4.9(1.1,22.1)$ in model 1 and $5.5(1.2,26.4)$ in model 2. For the 365-day mortality, a high RA was also associated with increased mortality (HR: $5.2,95 \%$ CI: 2.4, $11.3, \mathrm{p}$ for trend $<0.001)$. After adjusting for confounders, the association remained significant in model 1 (HR: 3.4, 95\% CI: 1.5, 7.6, $\mathrm{p}$ for trend $=0.003$ ) and model 2 (HR: 3.4, $95 \%$ CI: $1.5,7.7$, $\mathrm{p}$ for trend $=0.003$ ).

\section{Association Between RA and DKA-Related Infections}

Infections increase morbidity and mortality rates for ICU patients. Therefore, we investigated the association between
Table I Characteristics of Enrolled Patients

\begin{tabular}{|c|c|c|c|}
\hline Characteristics & $\begin{array}{l}\text { Low } \\
\text { Group }^{a}\end{array}$ & $\begin{array}{l}\text { High } \\
\text { Group }^{b}\end{array}$ & P-value \\
\hline $\mathrm{N}$ & 187 & 188 & \\
\hline Age & $42.7 \pm 15.6$ & $51.8 \pm 17.3$ & $<0.001$ \\
\hline $\begin{array}{l}\text { Gender, n (\%) } \\
\text { Male } \\
\text { Female }\end{array}$ & $\begin{array}{l}112(59.9) \\
75(40.1)\end{array}$ & $\begin{array}{l}106(56.4) \\
82(43.6)\end{array}$ & 0.491 \\
\hline $\begin{array}{l}\text { Vital signs } \\
\text { Heart rate, beats/minute } \\
\text { Respiratory rate, breaths/ } \\
\text { minute } \\
\mathrm{SBP}, \mathrm{mmHg} \\
\mathrm{DBP}, \mathrm{mmHg} \\
\mathrm{SPO}_{2}, \% \\
\text { Temperature, }{ }^{\circ} \mathrm{C}\end{array}$ & $\begin{array}{l}95.1 \pm 14.4 \\
18.8 \pm 3.5 \\
123.7 \pm 18.8 \\
65.3 \pm 12.6 \\
98.2 \pm 1.4 \\
36.9 \pm 0.4\end{array}$ & $\begin{array}{l}91.9 \pm 16.4 \\
19.9 \pm 4.4 \\
123.7 \pm 18.2 \\
62.1 \pm 11.1 \\
97.9 \pm 1.7 \\
36.8 \pm 0.7\end{array}$ & $\begin{array}{l}0.046 \\
0.008 \\
0.999 \\
0.009 \\
0.158 \\
0.082\end{array}$ \\
\hline $\begin{array}{l}\text { Comorbidities, } \mathrm{n}(\%) \\
\text { Congestive heart failure } \\
\text { Valvular disease } \\
\text { Renal failure } \\
\text { Chronic pulmonary } \\
\text { disease } \\
\text { Hypothyroidism } \\
\text { Liver disease } \\
\text { Sepsis } \\
\text { Septic shock }\end{array}$ & $\begin{array}{l}16(8.6) \\
1(0.5) \\
21(11.2) \\
22(11.8) \\
90(48.1) \\
15(8.0) \\
3(1.6) \\
3(1.6)\end{array}$ & $\begin{array}{l}40(21.3) \\
9(4.8) \\
59(31.4) \\
29(15.4) \\
109(58.0) \\
16(8.5) \\
21(11.2) \\
15(8.0)\end{array}$ & $\begin{array}{l}<0.001 \\
0.011 \\
<0.001 \\
0.301 \\
\\
0.056 \\
0.863 \\
<0.001 \\
0.004\end{array}$ \\
\hline $\begin{array}{l}\text { Laboratory parameters } \\
\text { Lactate, } \mathrm{mmol} / \mathrm{L} \\
\text { White blood cell, } \times 10^{9} / \mathrm{L} \\
\text { Albumin, g/L }\end{array}$ & $\begin{array}{l}2.0 \pm 1.2 \\
14.2 \pm 6.7 \\
4.2 \pm 0.6\end{array}$ & $\begin{array}{l}1.8 \pm 1.0 \\
14.0 \pm 7.7 \\
3.1 \pm 0.4\end{array}$ & $\begin{array}{l}0.193 \\
0.808 \\
<0.001\end{array}$ \\
\hline $\begin{array}{l}\text { 90-Day all-cause mortality, } \\
\text { n (\%) }\end{array}$ & $2(1.1)$ & $15(8.0)$ & 0.001 \\
\hline $\begin{array}{l}\text { 365-Day all-cause mortality, } \\
\text { n (\%) }\end{array}$ & $5(2.7)$ & $33(17.6)$ & $<0.001$ \\
\hline SAPSII & $27.8 \pm 10.7$ & $35.3 \pm 13.3$ & $<0.001$ \\
\hline SOFA & $2.8 \pm 1.9$ & $4.5 \pm 3.1$ & $<0.001$ \\
\hline
\end{tabular}

Notes: ${ }^{\text {a, b}}$ RA $<3.89$ was regarded as the low group, RA $>3.89$ was considered as the high group.

Abbreviations: RA, red blood cell distribution width-albumin ratio; SBP, systolic blood pressure; DBP, diastolic blood pressure; $\mathrm{SPO}_{2}$, oxygen saturation; SAPSII, The Simplified Acute Physiology Score II; SOFA, Sequential Organ Failure Assessment.

RA and DKA-related sepsis and septic shock (Table 3). RA was independently associated with sepsis (HR: $2.9,95 \%$ CI: 2.0, 4.1, p < 0.001) and septic shock (HR: 2.8, 95\% CI: 1.9, $4.2, \mathrm{p}<0.001$ ) in DKA patients. In model 1 , RA was still a significant predictor for sepsis (HR: 2.8, 95\% CI: 1.9, 4.1, $\mathrm{p}<0.001$ ) and septic shock (HR: 2.9, 95\% CI: 1.9, 4.3, p < 

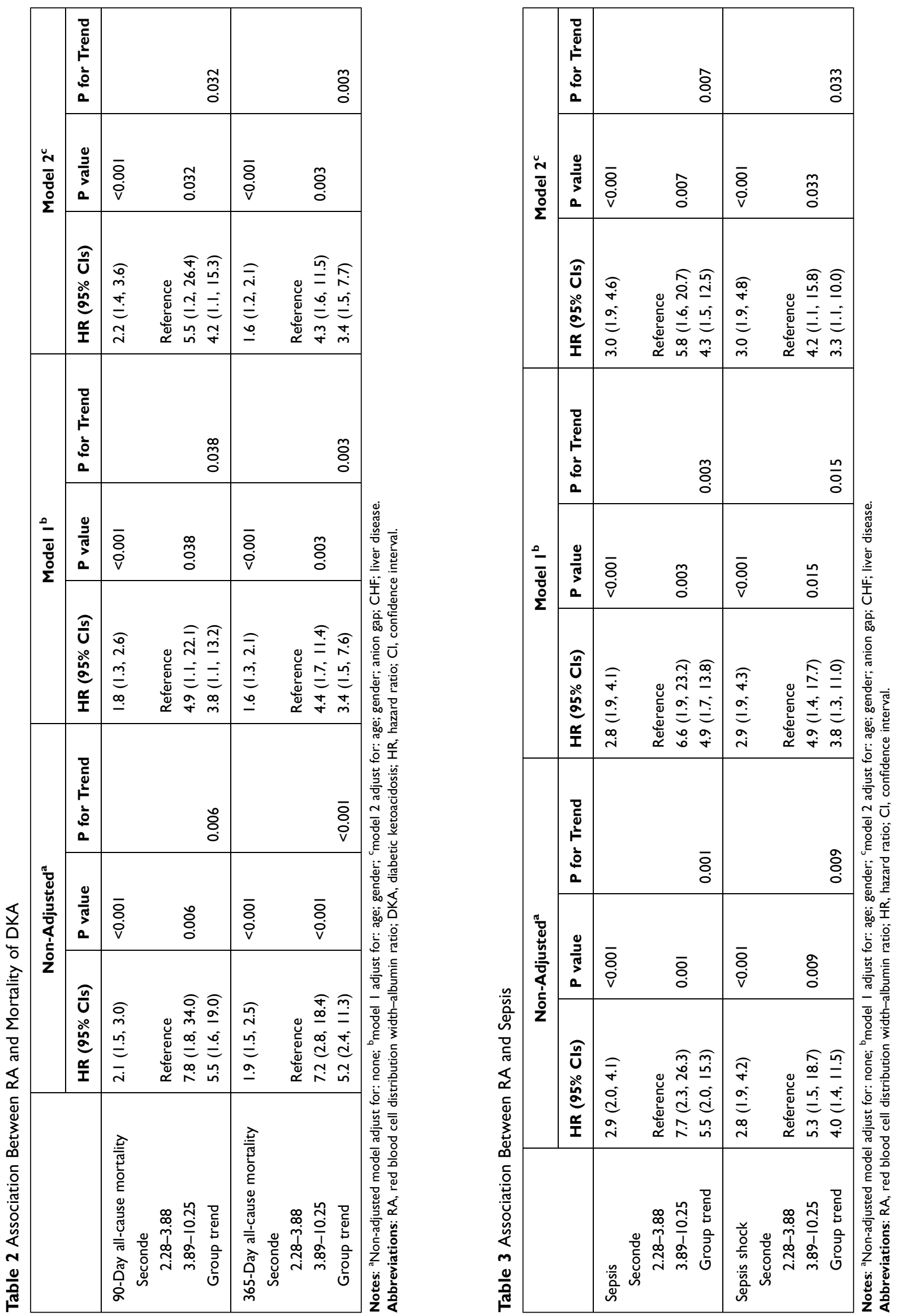
0.001). Model 2 revealed a similar result for sepsis (HR: 3.0, 95\% CI: 1.9, 4.6, p <0.001) and septic shock (HR: 3.0, 95\% CI: 1.9, 4.8, p<0.001). Moreover, in the non-adjusted model, a high RA was still found to be a predictor for an increased risk of sepsis (HR: 5.5, 95\% CI: 2.0, 15.3, p for trend $=0.001)$, model 1 (HR: $4.9,95 \% \mathrm{CI}: 1.7,13.8, \mathrm{p}$ for trend $=0.003$ ), and model 2 (HR: 4.3, 95\% CI: 1.5, 12.5, $\mathrm{p}$ for trend $=0.007)$. An increased RA indicated increased risk of septic shock in the non-adjusted model (HR: 4.0, 95\% CI: $1.4,11.5$, p for trend $=0.001)$, model 1 (HR: 3.8 , 95\% CI: 1.3, 11.0, p for trend $=0.015)$, and model $2(\mathrm{HR}$ : 3.3 , 95\% CI: $1.1,10.0, \mathrm{p}$ for trend $=0.033$ ).

\section{Discussion}

In this study, we found that RA was a predictor for allcause mortality in DKA patients, while an increased RA suggested an elevated risk of mortality. In addition, elevated RA was significantly associated with an increased risk of sepsis and septic shock in DKA patients.

RDW had the prognosis value for DKA. Dai et al reported that high RDW was related to an increased risk of all-cause mortality in DKA patients. ${ }^{14}$ While it was reported albumin could predict the occurrence of DKA, Karthikeyan et al had found that lower serum albumin level was associated with a high occurrence of DKA. ${ }^{15}$ They found that hypoalbuminemic DKA patients had higher level ketones in urine than normoalbuminemic DKA patients. This result was similar to our article. Although HR of RDW in DKA patients was increased in the above-cited article, the changes in variables were lower than those in our research. Yoo et al also reported that RA is better than RDW for predicting the mortality in acute respiratory distress syndrome. All results supported that RA might have more predict value than using RDW alone.

Zhang et al found RDW was useful predictor for sepsis. Sepsis patients with high value of RDW had higher all-cause mortality. ${ }^{16}$ The same trend was found in neonatal sepsis. ${ }^{17}$ Albumin also plays a predictive role in sepsis. Kendall et al found that low trend of serum albumin levels over time was associated with poor outcomes in patients with sepsis. ${ }^{18}$ It was also a strong predictor for sepsis in the elderly. ${ }^{19}$ All these results might partly explain the predicted role of RA in sepsis DKA patients.

The pathogenic mechanisms of DKA have not been clearly elucidated; however, it has been suggested that inflammation may play an important role in the process. ${ }^{6,7,20}$ High RDW levels and low albumin levels are strongly correlated with enhanced inflammation. ${ }^{10,21-25}$ Inflammatory cytokines lead to iron dysregulation, decreased erythropoietin production, damaged erythrocyte maturation, decreased RBC survival, enhanced immature erythrocyte circulation, and ultimately, increased anisocytosis. Albumin has the capacity for binding pro-inflammatory substances and decreasing inflammatory responses. In summary, a high RA, which represents elevated RDW and suppressed albumin levels, is a predictor for severe inflammation.

There are some limitations associated with this study. All data were obtained from the MIMIC III (v1.4) database; therefore, there may be a potential bias. Moreover, dynamic changes in RA, which may have elucidated on the predictive value of RA for DKA patients, were not evaluated in this study.

\section{Conclusions}

RA is a potential prognostic marker for DKA patients. A high RA is significantly correlated with increased allcause mortality of DKA and an increased incidence of DKA-related infections.

\section{Data Sharing Statement}

The raw data were available in figshare (dx.doi.org/ 10.6084/m9.figshare.15105195).

\section{Funding}

The authors declare that no funds were received to conduct this research.

\section{Disclosure}

The authors declare no conflicts of interest for this research.

\section{References}

1. Kitabchi AE, Umpierrez GE, Murphy MB, et al. Management of hyperglycemic crises in patients with diabetes. Diabetes Care. 2001;24(1):131-153. doi:10.2337/diacare.24.1.131

2. Modi A, Agrawal A, Morgan F. Euglycemic diabetic ketoacidosis: a review. Curr Diabetes Rev. 2017;13(3):315-321. doi:10.2174/ 1573399812666160421121307

3. Saeedi P, Petersohn I, Salpea P, et al. Global and regional diabetes prevalence estimates for 2019 and projections for 2030 and 2045: results from the international diabetes federation diabetes atlas, 9th) edition. Diabetes Res Clin Pract. 2019;157:107843. doi:10.1016/j. diabres.2019.107843

4. Cho NH, Shaw JE, Karuranga S, et al. IDF diabetes atlas: global estimates of diabetes prevalence for 2017 and projections for 2045. Diabetes Res Clin Pract. 2018;138:271-281. doi:10.1016/j. diabres.2018.02.023

5. Deshpande AD, Harris-Hayes M, Schootman M. Epidemiology of diabetes and diabetes-related complications. Phys Ther. 2008;88 (11):1254-1264. doi:10.2522/ptj.20080020 
6. Rains JL, Jain SK. Oxidative stress, insulin signaling, and diabetes. Free Radic Biol Med. 2011;50(5):567-575. doi:10.1016/j. freeradbiomed.2010.12.006

7. Li J, Huang M, Shen X. The association of oxidative stress and pro-inflammatory cytokines in diabetic patients with hyperglycemic crisis. J Diabetes Complications. 2014;28(5):662-666. doi:10.1016/j. jdiacomp.2014.06.008

8. Shen T, Braude S. Changes in serum phosphate during treatment of diabetic ketoacidosis: predictive significance of severity of acidosis on presentation. Intern Med J. 2012;42(12):1347-1350. doi:10.1111/ imj.12001

9. Li J, Shen X. Leptin concentration and oxidative stress in diabetic ketoacidosis. Eur J Clin Invest. 2018;48(10):e13006. doi:10.1111/ eci. 13006

10. Salvagno GL, Sanchis-Gomar F, Picanza A, et al. Red blood cell distribution width: a simple parameter with multiple clinical applications. Crit Rev Clin Lab Sci. 2015;52(2):86-105. doi:10.3109/10408363.2014.992064

11. Artigas A, Wernerman J, Arroyo V, et al. Role of albumin in diseases associated with severe systemic inflammation: pathophysiologic and clinical evidence in sepsis and in decompensated cirrhosis. J Crit Care. 2016;33:62-70. doi:10.1016/j.jcrc.2015.12.019

12. Fanali G, Di Masi A, Trezza V, et al. Human serum albumin: from bench to bedside. Mol Aspects Med. 2012;33:209-290. doi:10.1016/j. mam.2011.12.002

13. Yazici MU, Ayar G, Savas-Erdeve S, et al. Role of ischemia modified albumin serum levels as an oxidative stress marker in children with diabetic ketoacidosis. Comb Chem High Throughput Screen. 2019;22 (8):577-581. doi:10.2174/1386207322666191008214919

14. Dai H, Su X, Li H, et al. Association between red blood cell distribution width and mortality in diabetic ketoacidosis. J Int Med Res. 2020;48(3):300060520911494. doi:10.1177/0300060520911494

15. Karthikeyan J, Rajaragupathy S. Diagnostic usefulness of serum albumin as a predictor of diabetic ketoacidosis. Indian J Crit Care Med. 2018;22(10):733-736. doi:10.4103/ijccm.IJCCM_91_18

16. Zhang L, Yu $\mathrm{CH}$, Guo KP, et al. Prognostic role of red $\bar{b}$ blood cell distribution width in patients with sepsis: a systematic review and meta-analysis. BMC Immunol. 2020;21(1):40. doi:10.1186/s12865020-00369-6
17. Martin SL, Desai S, Nanavati R, et al. Red cell distribution width and its association with mortality in neonatal sepsis. J Matern Fetal Neonatal Med. 2019;32(12):1925-1930. doi:10.1080/ 14767058.2017.1421932

18. Kendall H, Abreu E, Cheng AL. Serum albumin trend is a predictor of mortality in ICU patients with sepsis. Biol Res Nurs. 2019;21 (3):237-244. doi:10.1177/1099800419827600

19. Arnau-Barres I, Guerri-Fernandez R, Luque S, et al. Serum albumin is a strong predictor of sepsis outcome in elderly patients. Eur J Clin Microbiol Infect Dis. 2019;38(4):743-746. doi:10.1007/s10096-01903478-2

20. Kim F, Tysseling KA, Rice J, et al. Free fatty acid impairment of nitric oxide production in endothelial cells is mediated by IKKbeta. Arterioscler Thromb Vasc Biol. 2005;25(5):989-994. doi:10.1161/01. ATV.0000160549.60980.a8

21. McMillan DC. Systemic inflammation, nutritional status and survival in patients with cancer. Curr Opin Clin Nutr Metab Care. 2009;12 (3):223-226. doi:10.1097/MCO.0b013e32832a7902

22. Mukai H, Villafuerte H, Qureshi AR, et al. Serum albumin, inflammation, and nutrition in end-stage renal disease: C-reactive protein is needed for optimal assessment. Semin Dial. 2018;31(5):435-439. doi:10.1111/sdi.12731

23. Eckart A, Struja T, Kutz A, et al. Relationship of nutritional status, inflammation, and serum albumin levels during acute illness: a prospective study. Am J Med. 2020;133(6):713-722 e717. doi:10.1016/j.amjmed.2019.10.031

24. Bozkurt E, Ucak T. Serum inflammation biomarkers in patients with keratoconus. Ocul Immunol Inflamm. 2020:1-4. doi:10.1080/ 09273948.2020.1741648

25. Cintesun E, Akar S, Gul A, et al. Subclinical inflammation markers in hyperemesis gravidarum and ketonuria: a case-control study. J Lab Physicians. 2019;11(02):149-153. doi:10.4103/JLP.JLP_151_18
International Journal of General Medicine

\section{Publish your work in this journal}

The International Journal of General Medicine is an international, peer-reviewed open-access journal that focuses on general and internal medicine, pathogenesis, epidemiology, diagnosis, monitoring and treatment protocols. The journal is characterized by the rapid reporting of reviews, original research and clinical studies across all disease areas. The manuscript management system is completely online and includes a very quick and fair peer-review system, which is all easy to use. Visit http://www.dovepress.com/ testimonials.php to read real quotes from published authors. 